\title{
Protocol for the Evaluation of Aborted Fetuses and Stillborn Infants in the Delivery Room
}

\begin{abstract}
Objectives: Congenital malformations are a common cause of intrauterine death and represent one of the most frequent indication for induced abortion. A multidisciplinary approach for the clinical evaluation of aborted fetuses and stillborn infants is a fundamental step in order to identify the cause of congenital anomalies and ensure an appropriate parental counseling in terms of recurrence risk and primary and secondary prevention for future pregnancies
\end{abstract}

The aim of this study was to design and test a diagnostic protocol for the clinical evaluation of aborted fetuses and stillborn infants to be applied in the delivery room.

Study design: This is a prospective study including stillbirths, induced abortions and miscarriages occurred during a 10-months period in the Gynecology and Obstetrics Unit of the University Hospital of Padova, Italy. The diagnostic protocol, applied by a clinical geneticist, consisted of the following 4 parts: collection of an accurate 3 -generation family history and parental medical history; external physical examination of the fetus; taking photographs for future reference; $\mathrm{X}$-ray evaluation and additional analyses in selected cases.

Results: The proposed diagnostic protocol was applied in 18 miscarriages, 42 terminations of pregnancy and 11 intrauterine fetal deaths.

A chromosomal anomaly had been prenatally diagnosed by invasive diagnostic procedures (chorionic villus sampling or amniocentesis) in 21 fetuses. Isolated or multiple anomalies had been detected by ultrasound in 22 fetuses without the prenatal diagnosis of a chromosomopathy. The external physical examination identified additional findings in $33 \%$ of cases with isolated anomalies and in all the cases with multiple malformations detected by the prenatal ultrasound evaluation. The application of the protocol allowed to diagnose a specific genetic disease in $50 \%$ of fetuses with multiple congenital defects.

Conclusion: The diagnostic protocol proposed in this study is reliable, simple, low-cost and can be applied in any hospital. Neonatologists and midwives are always present in the delivery room and can easily collect essential data that can be subsequently discussed with a team of specialists also through teleconsultation.

Keywords: Midwives; Neonatologists; Stillbirth; Spontaneous abortion; Termination of pregnancy; Congenital anomalies
Volume 8 Issue I - 2018

\author{
Matteo Cassina,' Elena Di Gianantonio,' \\ Yasmin Al Naber,' Anna Gazzola Martini, ${ }^{2}$ \\ Marta Dona,' Maria Teresa Gervasi, ${ }^{3}$ \\ Giovanni Battista Nardelli, ${ }^{2}$ Maurizio \\ Clementi'
}

'Clinical Genetics Unit, Department of Women's and Children's Health, University of Padova, Padova, Italy

2Obstetrics and Gynecology Clinic, Department of Women's and Children's Health, University of Padova, Padova, Italy ${ }^{3}$ Obstetrics and Gynecology Clinic, Hospital of Padova, Padova, Italy

Correspondence: Maurizio Clementi, Genetica Clinica ed Epidemiologica, Dipartimento di Salute della Donna e del Bambino,Via Giustiniani 3, 35 I 28 Padova, Italy, Tel 39 04982। 3572, Fax $39-4982$ I I 425, Email maurizio.clementi@unipd.it

Received: November 08, 2017| Published: January 09, 2018

\section{Introduction}

Congenital malformations are not uncommon findings in human pregnancies and their correct morphologic and etiologic classification is essential to ensure an appropriate counseling for the couple in terms of recurrence risk and primary and secondary prevention for future pregnancies.

Congenital defects may be isolated or multiple and represent a relevant cause of spontaneous abortion and fetal death when they are incompatible with the normal embryo-fetal development. ${ }^{1}$ Furthermore, the routine prenatal ultrasound examination performed in many countries allows the detection of several major fetal defects and pregnancies are frequently terminated if the fetus is severely affected. ${ }^{2}$

The systematic collection of relevant information regarding the family, personal and obstetric histories and the fetal evaluation after a miscarriage, stillbirth and termination of pregnancy are fundamental steps to reach a specific diagnosis. Formal protocols have been developed for stillbirth examination in Canada (Alberta Perinatal Health Program, Perinatal Services British Columbia),
USA (Wisconsin Stillbirth Service Program, Stillbirth Collaborative Research Network), and Australia/New Zealand (Perinatal Society of Australia and New Zealand - Clinical Practice Guideline for Perinatal Mortality)..$^{3-7}$

According to the literature, the external physical examination of the fetus adds important information in the diagnostic process in a proportion highly variable from 25 to $90 \%$ of cases. ${ }^{1,8}$ In addition, the external physical examination and the autopsy of fetuses are essential to confirm all the defects prenatally detected and to identify associated anomalies that could be missed or be undetectable at the ultrasound examination. However, although clinical practice guidelines have been proposed to standardize the investigation, a systematic fetal examination in case of stillbirth, miscarriage and termination of pregnancy is not performed in most Obstetrics and Gynecology Units. ${ }^{7}$

Ideally, a multidisciplinary team of experienced physicians including gynecologists, neonatologists, clinical geneticists and perinatal pathologists should perform the examination, but a 24-hour specialist consultation is not available in most hospitals. However, neonatologists and midwives are always present in the delivery room and would be able to apply a diagnostic protocol and perform 
a systematic evaluation of the fetus; all the collected data could be subsequently discussed with other specialists.

The aim of this study is to test and propose a diagnostic and standardized protocol for the clinical evaluation of aborted fetuses and stillborn infants that could be performed in the delivery room.

\section{Materials and Methods}

This prospective observational study assessed 71 consecutive aborted fetuses and stillborn infants delivered during a 10-months period in the Gynecology and Obstetrics Unit of the University Hospital of Padova. Induced abortions after the prenatal diagnosis of chromosomal anomalies or congenital malformations were included. Spontaneous abortions prior to the 14th gestational week were excluded. Each fetus was examined according to a pre-designed protocol applied by a midwife (A.G.M.) tutored by a clinical geneticist (M.D, E.D.G, M.Cl).

\section{The protocol consisted of the following parts}

Collection of data regarding family and parental medical history: A three-generation family history, the complete parental medical history and the obstetric history were recorded using a standardized form. Data included any maternal condition complicating the pregnancy such as chronic disorders, maternal infections and exposure to teratogens; in addition, the results of both invasive and non-invasive prenatal analyses were also carefully collected (i.e. fetal ultrasound evaluations, screening tests for Down syndrome, chorionic villus sampling and amniocentesis).

External physical examination of the fetus: The external physical examination of the fetus was performed in all cases. External measurements (weight, crown-heel length and occipital frontal circumference) and a detailed description of dysmorphic features, external malformations/deformations and pertinent negative findings were recorded. The form is available upon request.

Clinical photographs:Pictures of the fetus from several views were collected in all cases for future reference and included whole body frontal photos, frontal and lateral pictures of the face, and photos of any abnormal parts

X-ray evaluation: Skeletal X-ray examinations were performed only in selected cases to further evaluate suspected skeletal abnormalities (such as limb defects, disproportionate upper limb/lower limb ratio or other physical deformities).

In order to evaluate the applicability of the protocol in peripheral hospitals and its accuracy, photographs of all fetuses were remotely examined by a clinical geneticist (M.Ca.) who had not participated at the live examinations. Then, the results of live and remote examinations were compared.

\section{Results}

In the study period, 18 miscarriages, 42 terminations of pregnancy and 11 intrauterine fetal deaths were evaluated. The diagnostic protocol was applied in 69 cases, while it could not be completed for two miscarried fetuses because of their marked autolysis.

A prenatal invasive test had been performed in 43 cases $(60.6 \%)$ and a pathologic chromosomal anomaly had been diagnosed in 21 fetuses.

The second trimester fetal ultrasound examination had detected congenital malformations, deformations, growth retardation or other anomalies in 33 fetuses (46.5\%). After the exclusion of 11 cases with congenital defects caused by chromosomal abnormalities, an isolated anomaly had been diagnosed in 18 fetuses while multiple defects had been detected in the remaining 4 cases; no etiologic diagnoses had been hypothesized or confirmed in these 22 malformed fetuses. The anomalies detected before the application of the protocol are listed in Table 1.

Table I Anomalies detected during pregnancy

\begin{tabular}{ll}
\hline Chromosomal anomalies $(\mathbf{n})$ & $\mathbf{2 1}$ \\
\hline Trisomy 21 & 17 \\
Trisomy 18 & $\mathrm{I}$ \\
Submicroscopic chromosomal abnormality & $\mathrm{I}$ \\
Mosaic sex chromosome aneuploidy & $\mathrm{I}$ \\
Triploidy & $\mathrm{I}$ \\
Fetal abnormalities with unknown cause (n) & $\mathbf{2 2}$ \\
Isolated aomalies & 18 \\
Multiple malformations & 4 \\
No anomalies $(\mathbf{n})$ & $\mathbf{2 8}$ \\
\hline
\end{tabular}

The external physical examination provided additional minor findings in 33\% of cases with isolated congenital malformations and in all the 4 fetuses with multiple congenital anomalies. In the latter group the protocol was fundamental for a revision of the diagnosis. In one fetus with prenatal detection of limb reduction, the final diagnosis of thanatophoric dysplasia was given on the basis of the external evaluation and the skeletal radiological examination. In another male fetus with prenatal detection of agenesis of the corpus callosum and anomalies of the tongue, the external evaluation identified a preaxial polydactyly suggesting the diagnosis of Acrocallosal syndrome.

The results of the external physical examinations of fetuses with isolated or multiple anomalies prenatally detected by ultrasound are reported in Table 2.

After a short training period, the midwife was able to accurately collect the information required by the diagnostic protocol, to perform a first-level external evaluation of fetuses and to document adequately any morphologic anomaly with photographs.

In all cases, the remote blinded revaluation of photographs and medical records has come to the same conclusions reached after the live examination.

\section{Comment}

The external examination and the autopsy of spontaneously or voluntarily aborted fetuses and stillborn infants are essential to confirm malformations diagnosed by ultrasound during pregnancy and to identify any other undetected external anomaly. The sensitivity of second trimester ultrasound in the detection of fetal anomalies has been reported to range from $13.5 \%$ to $87.5 \%$ according to the protocol and the sampling of cases used in different studies. ${ }^{2,9}$ in addition, the external physical examination of fetuses, performed after the delivery, has been reported to identify additional abnormalities in at least $25 \%$ of cases. ${ }^{3,10}$

The etiologic classification is essential to ensure an adequate counseling for the couple in terms of recurrence risk and primary and secondary prevention for future pregnancies. In fact, the recurrence risk varies according to the etiology of the malformation, that can be genetic (single gene mutations or chromosomal abnormalities), environmental (mechanical constraints on fetal development, infections, maternal diseases, drugs, chemicals and radiation exposure) or multifactorial. Most of stillborn infants presenting with congenital malformation are affected by genetic disorders. ${ }^{11,12}$ 
Table 2 Results of the external physical examination of fetuses with isolated or multiple anomalies prenatally detected by ultrasound (cases with chromosomal abnormalities are excluded).

\begin{tabular}{|c|c|c|c|}
\hline Anomalies prenatally detected & External physical examination after delivery & TOP/ IUFD & g.w. \\
\hline \multicolumn{4}{|l|}{ Single anomaly $(n=18)$} \\
\hline Corpus Callosum Agenesis & Micrognathia and Left clubfoot & TOP & 21 \\
\hline Bilateral Renal Agenesis & External examination not performed due to marked fetal autolysis & IUFD & 35 \\
\hline $\begin{array}{l}\text { Absence of the Septum Pellucidum, } \\
\text { Aqueductal stenosis and bilateral clubfoot }\end{array}$ & $\begin{array}{l}\text { Sacral dimple, bilateral clubfoot, } \\
\text { mild facial dysmorphism }\end{array}$ & TOP & 23 \\
\hline Bilateral Renal Agenesis & Hypertelorism, micrognathia, flat face & TOP & 16 \\
\hline $\begin{array}{l}\text { Mild cardiomegaly with atrioventricular } \\
\text { valvular insufficiency }\end{array}$ & No anomalies & TOP & 22 \\
\hline Hypoplastic left heart and cystic hygroma & Fetal hydrops and cystic hygroma & TOP & 16 \\
\hline Hypoplastic left heart & Bilateral clubfoot & TOP & 22 \\
\hline $\begin{array}{l}\text { Spina Bifida and } \\
\text { Arnold-Chiari malformation }\end{array}$ & Spina Bifida and left clubfoot & TOP & 17 \\
\hline Diaphragmatic Hernia & No anomalies & TOP & 22 \\
\hline Hydrops & Hydrops & TOP & 19 \\
\hline Hydrops & Hydrops and bilateral clubfoot & TOP & 21 \\
\hline IUGR & $\begin{array}{l}\text { External examination not performed } \\
\text { due to marked fetal autolysis }\end{array}$ & IUFD & 24 \\
\hline IUGR & Hypertelorism & IUFD & 24 \\
\hline $\begin{array}{l}\text { Megacystis with dilatation of } \\
\text { ureters and renal pelvis }\end{array}$ & Facial asymmetry and hypoplastic left ear & TOP & 17 \\
\hline $\begin{array}{l}\text { Polyhydramnios, stomach not } \\
\text { visualized: suspected esophageal atresia }\end{array}$ & $\begin{array}{l}\text { Micrognathia and single } \\
\text { palmar crease (right hand) }\end{array}$ & IUFD & 37 \\
\hline $\begin{array}{l}\text { Corpus Callosum Agenesis and } \\
\text { partial Holoprosencephaly }\end{array}$ & $\begin{array}{l}\text { No external anomalies } \\
\text { associated with holoprosencephaly }\end{array}$ & TOP & 22 \\
\hline $\begin{array}{l}\text { Severe ventriculomegaly } \\
\text { with cerebella hypoplasia }\end{array}$ & Short neck & TOP & 21 \\
\hline Mild ventriculomegaly & Micrognathia & TOP & 22 \\
\hline \multicolumn{4}{|l|}{ Multiple anomalies $(n=4)$} \\
\hline $\begin{array}{l}\text { Corpus Callosum Agenesis, } \\
\text { Retrognathia,Tongue anomalies }\end{array}$ & $\begin{array}{l}\text { Retrognathia, retroposition of the } \\
\text { tongue, preaxial polydactyly (suspected Acrocallosal syndrome) }\end{array}$ & TOP & 22 \\
\hline $\begin{array}{l}\text { Skeletal dysplasia, } \\
\text { reduced skull ossification }\end{array}$ & Undefined Skeletal Dysplasia & TOP & 16 \\
\hline Bilateral cleftlip, agenesis of right kidney & Minor anomalies & TOP & 22 \\
\hline Narrow thorax, short limbs & $\begin{array}{l}\text { Short limbs (total body X-ray imaging), } \\
\text { unilateral clubfoot, minor facial anomalies (Thanatophoric } \\
\text { dysplasia) }\end{array}$ & TOP & 22 \\
\hline
\end{tabular}

IUFD: Intrauterine Fetal Demise;TOP:Termination of Pregnancy; gw: Gestational Week

Our study confirmed the usefulness of a standardized data collection and of the external physical evaluation of the fetus after the delivery; in fact, excluding cases with prenatal diagnosis of chromosomal abnormality, our examinations allowed to observe additional minor findings, for which the sensitivity of the ultrasound evaluations is very low, in $33 \%$ of cases with an isolated major malformation. In addition, the external physical examination added useful information in all the 4 cases with multiple anomalies prenatally detected by ultrasound. In particular, in 2 cases the application of the protocol allowed to yield a specific diagnosis that otherwise would have been missed and to provide the couple with a precise recurrence risk for future pregnancies.

The fetal evaluation should ideally be performed by a multidisciplinary team of specialists with experience in fetal pathology, but this is not always possible in several peripheral hospitals, where a 24-hour specialist consultation is not available. It is therefore necessary to identify other professionals that are able to collect essential data and perform a first-level examination of the fetus following a standardized protocol such as the one we have proposed. The collected data can be subsequently discussed with a team of specialists.
The best candidates for this task are neonatologists and midwives; they are always present in the delivery room and have established skills in the collection of information regarding pregnant women's medical history and in performing the external physical evaluation of newborns. In our experience, after a short training period, the midwife was able to perform accurately the diagnostic protocol we have proposed.

It is important to note that in our protocol the photographs represent an irreplaceable integration of the clinical external examination. In fact, they provide a permanent record of the macroscopic morphology of the fetus for later interpretation. We have evaluated the reliability of the remote external fetal evaluation by photos and have observed that it effectively allows to recognize most of the external abnormalities that had been previously detected during the live evaluation.

Our protocol could be integrated with the collection and conservation of fetal tissues in order to extract the DNA and perform the array-CGH analysis or targeted genetic tests in case a specific genetic disease is suspected. Cord blood could be collected after the delivery; alternatively, a sample of fetal blood $(1-3 \mathrm{~mL})$ could be collected by cardiac puncture within few hours after the delivery. 
To perform genetic tests, DNA could be obtained also from skin, umbilical cord or internal tissues; samples should be stored frozen and not fixed.

In conclusion, the identification of the etiology of malformations in stillborn and aborted fetuses is fundamental to provide the couple with a correct counseling regarding the recurrence risk, to identify preventable causes and to improve the management in subsequent pregnancies. The diagnostic protocol proposed in this study is reliable, simple, low-cost and can be applied in any hospital. Midwives and neonatologists are always present in the delivery room and can easily collect essential data that can be subsequently discussed with a team of specialists also through teleconsultation.

\section{Conflict of interest statement}

The authors declare no conflicts of interest.

\section{References}

1. Silver RM, Varner MW, Reddy U, Goldenberg R, Pinar H, et al. (2007) Work-up of stillbirth: a review of the evidence. Am J Obstet Gynecol 196(5): 433-444.

2. Clementi M, Stoll C (2001) The Euroscan study. Ultrasound Obstet Gynecol 18(4): 297-300.

3. Corabian P, Scott NA, Lane C, Guyon G (2007) Guidelines for investigating stillbirths: an update of a systematic review. J Obstet Gynaecol Can 29(7): 560-567.

4. Dudley DJ, Goldenberg R, Conway D, Silver RM, Saade GR, et al. (2010) A new system for determining the causes of stillbirth. Obstet Gynecol 116(2 Pt 1): 254-260.
5. Flenady V, Mahomed K, Ellwood D, Charles A, Teale G, et al. (2010) Uptake of the Perinatal Society of Australia and New Zealand perinatal mortality audit guideline. Aust N Z J Obstet Gynaecol 50(2): 138-143.

6. Parker CB, Hogue CJ, Koch MA, Willinger M, Reddy UM, et al (2011) Stillbirth Collaborative Research Network: design, methods and recruitment experience. Paediatr Perinat Epidemiol 25(5): 425-435.

7. Vander Wielen B, Zaleski C, Cold C, McPherson E (2011) Wisconsin stillbirth services program: a multifocal approach to stillbirth analysis. Am J Med Genet A 155A (5): 1073-1080.

8. Pauli RM, Reiser CA (1994) Wisconsin Stillbirth Service Program: II. Analysis of diagnoses and diagnostic categories in the first 1,000 referrals. Am J Med Genet 50(2): 135-153.

9. Grandjean H, Larroque D, Levi S (1999) The performance of routine ultrasonographic screening of pregnancies in the Eurofetus Study. Am J Obstet Gynecol 181(2): 446-454

10. Pinar H, Koch MA, Hawkins H, Heim-Hall J, Abramowsky CR, et al. (2012) The stillbirth collaborative research network postmortem examination protocol. Am J Perinatol 29(3): 187-202.

11. Korteweg FJ, Bouman K, Erwich JJ, Timmer A, Veeger NJ, et al.(2008) Cytogenetic analysis after evaluation of 750 fetal deaths: proposal for diagnostic workup. Obstet Gynecol 111(4): 865-874.

12. Fretts RC (2005) Etiology and prevention of stillbirth. Am J Obstet Gynecol 193(6): 1923-1935. 